\title{
Estimación del nivel de desarrollo sostenible de los territorios rurales del área de biosfera macizo del cajas, a partir de las dimensiones del sistema territorial
}

\section{Estimation of the level of sustainable development of rural territories in the solid biosphere area of the cajas, based on the dimensions of the territorial system}

\author{
Noé Alberto Carchipulla Zhunio ${ }^{1 *}$ \\ ${ }^{1}$ Secretaría Nacional de Planificación y Desarrollo \\ *ncarchipulla@senplades.gob.ec \\ DOI: https://doi.org/10.26871/killkana_social.v2i3.320
}

\begin{abstract}
Resumen
El problema que conduce la investigación, refiere a los vacíos de información cuantitativa y operativa para análisis del nivel de desarrollo sostenible de los territorios rurales del Área de Biosfera Macizo del Cajas, y en consecuencia no permiten tomar decisiones pragmáticas que encaminen a la sostenibilidad. Estos vacíos de data resulta un imperativo, aún más cuando el modelo de la reserva de biosfera apunta a los principios del desarrollo sostenible. Ante la situación planteada el objetivo marco se determina en el análisis de contraste del nivel de sostenibilidad alcanzado por los gobiernos sub-nacionales rurales pertenecientes a la Biosfera Macizo del Cajas para identificar el grado de desempeño, los desequilibrios entre dimensiones, como patrones de desarrollo territorial, teniendo en cuenta la multidimensionalidad del sistema territorial. En relación directa a los objetivos de la investigación, el autor aplica como marco metodológico para la estimación del nivel de desarrollo sostenible de los territorios rurales del Área de Biosfera Macizo del Cajas (ABMC) la herramienta denominada Índice de Desarrollo Sostenible. En efecto el recurso metodológico y heurístico empleado demanda la elección propia de indicadores de las dimensiones del sistema territorial y que correspondan a las unidades de observación y análisis expresada en los territorios rurales del espacio denominado Biosfera, para esto será preciso iniciar con la construcción de información base que modele este territorio, para seguidamente insumirlo a la metódica de aplicación. Los resultados luego de un tratamiento posterior de los datos determinaron una estimación del nivel desarrollo sostenible del ABMC con un nivel de estado del sistema catalogado como inestable, el valor del Índice es 0,51, así mismo, se determina territorios con un nivel crítico, inestable y estable.
\end{abstract}

Palabras clave: biosfera, desarrollo sostenible, sistema territorial, territorio.

\begin{abstract}
The problem leading the investigation, refers to the gaps in quantitative and operational information for analysing the level of sustainable development of the rural territories of the solid biosphere area of the Cajas, and therefore do not allow to make decisions Pragmatics that will be a step for sustainability. These data gaps are imperative, even more so when the biosphere reserve model points to the principles of sustainable development. In view of the situation posed the framework objective is determined in the contrast analysis of the level of sustainability achieved by the sub-national governments belonging to the solid biosphere of the boxes to identify the degree of performance, Imbalances between dimensions, as patterns of territorial development, taking into account the multidimensionality of the territorial system. In direct relation to the objectives of the investigation, the author applies as methodological framework for the estimation of the level of sustainable development of the rural territories of the area of solid biosphere of the boxes (ABMC) the tool called Index of development Sustainable. In fact, the methodological and heuristic resource used demands the choice of indicators of the dimensions of the territorial system and corresponding to the units of observation and analysis expressed in the rural territories of the space called Biosphere, for this it will be necessary to initiate with the construction of base information that model this territory, then to summarize it to the methodical of application. The results after a subsequent treatment of the data determined an estimate of the level sustainable development of the ABMC with a level of state of the system classified as unstable, the value of the index is 0.51, likewise, it is determined territories with a level Critical, unstable and stable.
\end{abstract}

Key words: Biosphere, sustainable development, territorial system, territory. 


\section{Introducción}

La investigación se desarrolla en un espacio denominado Área de Biosfera Macizo del Cajas (ABMC) donde por definición responde a los preceptos de Desarrollo Sostenible. Por tanto el autor utiliza como recurso metodológico y heurístico el estudio de los procesos de desarrollo territorial, identificando como unidad de análisis a los territorios rurales, basado en indicadores para la caracterización y ordenación del territorio.

Lo anterior apunta a estimar el nivel de desarrollo sostenible de los territorios rurales del Área de Biosfera Macizo del Cajas (ABMC) para identificar el grado de desempeño, los desequilibrios como patrones de desarrollo territorial, teniendo en cuenta múltiples dimensiones del sistema territorial, que deriva a objetivos complementarios, para tal propósito, a saber: 1) construir una base de datos estadística como línea base del ABMC a partir de la información del VII Censo de Población y VI de Vivienda 2010 contemplada en información de población, vivienda, hogar, insumo intermedio, para la obtención de variables e indicadores a nivel de las dimensiones social, económica y ambiental y que sean representativos del desarrollo sostenible; 2) Expandir la base de datos construida del ABMC a nivel del esquema de ordenamiento marco establecido de la Reserva de Biosfera, esto es zonación: núcleo, amortiguamiento y transición; y 3 ) Establecer indicadores que representan a cada una de las dimensiones del desarrollo sostenible en las unidades territoriales rurales, para el cálculo del índice de Desarrollo sostenible.

Este planteamiento de la investigación se justifica porque para la detección y estudio de los problemas que debe atender y prever la ordenación territorial contemplada en el corpus conceptual de desarrollo sostenible debe caracterizar al territorio de forma multidimensional orientados al desarrollo, es decir, comparar dentro de cada componente del sistema, territorios que presenten rezagos, y grados de desempeño no acordes, así mismo, porque por prelación identificará espacialmente que territorios rurales del ABMC presentan grados de diferenciación y similitud a nivel de la dimensión ambiental, económica y social, reflejada por variables que estructuran el sistema territorial. También porque la investigación se relaciona con las prioridades de la región y del país entorno a los convenios marco establecidos (Marco Estatutario y Estrategia de Sevilla) que se comprometió el Estado a cumplirlos referente a la gestión de las Reservas Biosfera, donde recalco, el objetivo principal II: Utilizar las Reservas de Biosfera como Modelo en la Ordenación del Territorio y Lugares de Experimentación del Desarrollo Sostenible.

Otro argumento radica en los resultados, estos se utilizarán para brindar conocimientos a los estudios relacionados a la temática, y para replicar el estudio a un contexto similar (otras RB del País).

Desde el punto de vista operacional se definieron indicadores para la caracterización y ordenación del territorio. Estos indicadores se estructuran en tres dimensiones: So- cial, Económica y Ambiental. Sobre dichos indicadores se ha llevado a cabo un promedio aritmético en cada territorio y de forma agregada al ABMC, esta expresión matemática dimensional se la realiza a través del índice Integrado de Desarrollo Sostenible establecido por Sepúlveda (2008). En total, se han seleccionado 26 indicadores de Desarrollo Sostenible cuantificado para 51 territorios rurales de la Reserva de Biosfera. El resultado lleva a que los territorios rurales se puedan describir no solo a través de la integralidad de los indicadores, sino también en función de cada dimensión.

El presente documento se estructura en cinco acápites, siendo el primero esta introducción. La segunda parte avanza a establecer la matriz teórica que sustenta la investigación, y luego se expone como recurso metodológico el Índice de desarrollo sostenible, a la par se precisa las técnicas y los instrumentos que el autor empleo para la construcción de información estadística de línea base para el territorio. En el cuarto acápite se procede a la estimación del nivel de desarrollo sostenible de los territorios rurales del Área de Biosfera Macizo del Cajas, y finalmente las conclusiones y recomendaciones.

\section{Marco Teórico}

Propende entonces delimitar y acotar el objeto de estudio mediante un examen conceptual. La idea subyace en determinar la acepción de desarrollo sostenible en territorios rurales, empero, se resalta como ejes conceptuales al de Desarrollo Sostenible, el Enfoque Territorial Rural y la Reserva de Biosfera.

\subsection{Conceptos, definiciones e interrelaciones}

En concreto, Desarrollo Sostenible (DS) lo concebimos como base de la planificación territorial, "porque es en términos de calidad de vida, como la ordenación del territorio entiende el desarrollo" (Gómez y Gómez, 2013), así, la sostenibilidad humana implica dimensiones del desarrollo como estructura para consolidar ese fin, a propósito Leenders y De Leus (2009) declaran. "El referente conceptual de la planificación en general es el del desarrollo humano sostenible (...)" (p.1). Según se ha citado y apegados a la definición de legado sobre DS establecido por la Comisión Brutland ${ }^{1}$ en los años 80, se extrae el término necesidades, a razón, Bioret, Cibien, Grénot y Lecomte, 1998 sostienen. "una actividad humana contribuye al desarrollo sustentable cuando responde a las siguientes cuatro necesidades: materiales, sociales, calidad ambiental y equidad (...)" (p. 29), simultáneamente se relacionan también con la acepción que el enfoque territorial plasma del DS. "El desarrollo se concibe como un proceso multidimensional e inter-temporal, enmarcado en una cuadriga cuyos ejes son la equidad, la sostenibilidad, la competitividad y la gobernabilidad" (Sepúlveda, 2008). Lo podemos referir como un metaconcepto por los elementos

\footnotetext{
1 "Satisfacer las necesidades de las generaciones presentes sin comprometer las posibilidades de las del futuro para atender sus propias necesidades" (Nuestro Futuro Común. 1987).
} 
que aborda. Por consiguiente las dimensiones del desarrollo sostenible, que también son dimensiones del territorio, conforman la integralidad de este último. De manera que no es lo social, lo ambiental o lo económico que son sustentables, sino todo el sistema territorial en su conjunto, se debe agregar que sin una planificación sostenible y con visión territorial, los frutos del desarrollo se agrupan en uno o varios territorios y no de forma equilibrada en todos.

Basados en el estamento mayor de DS y su adaptación a una definición más contemporánea que denota su adaptabilidad e integración al territorio, surge el Desarrollo Rural Sostenible (DRS) que enfoca su análisis para atender una diversidad de vicisitudes del área rural (pobreza, agricultura), para ello postula el enfoque territorial fundamentado en el desarrollo micro regional y la nueva ruralidad, ambos nacidos en la década de los noventa (Sepúlveda, Rodríguez, Echeverri \& Portilla, 2003). Estos mismos autores lo definen así:

El enfoque territorial descrito en este libro plantea como principio la necesidad de superar la concepción del desarrollo rural como asistencia social, para establecerlo como una verdadera estrategia de desarrollo, que integra efectivamente las articulaciones macro y sectoriales en espacios territoriales, con la meta de lograr mayor cohesión social y territorial. (p.7)

Seguidamente relacionamos un elemento deliberante que es imprescindible no dejar de mencionarlo, el territorio. La síntesis conceptual apunta a la notación de territorio como un espacio subnacional como lo establece Castro (2015) “(...) definida la Organización Territorial del Estado, a cada territorio de la misma le corresponde un determinado Gobierno Autónomo Descentralizado con sus respectivas competencias exclusivas, algunas de las cuales tienen una directa incidencia en el ordenamiento territorial". (p.16). Así mismo, esos territorios son caracterizados por una multidimensionalidad de criterios propios y distintivos de los espacios rurales (Schneider y cols., 2007), y que en coherencia con la metodología a abordar, el fin mismo que se presenta es la gestión de los territorios rurales, a lo que el autor lo define como la unidad básica de observación y análisis.

De nuevo se resalta que de acuerdo al enfoque territorial de desarrollo rural sostenible dentro de sus elementos imperativos, la multidimensionalidad abarca componentes del sistema territorial: a) dimensión económica; b) dimensión social; c) dimensión ambiental; d) dimensión político institucional, elemento que son propios de los territorios y que permite su relacionamiento.

En esta misma línea, Gómez (2013) establece que los componentes del sistema territorial constituyen el medio físico natural, la población, actividades, asentamientos humanos, infraestructuras o canales de relación y el marco institucional y legal, su flexibilidad se adecua a diferentes espacios territoriales como la reserva de Biosfera.
Según Falconí (2004) "La noción de desarrollo sostenible es multidimensional", nos enfrentamos entonces a decisiones con alcance en diferentes aristas, no tratamos o enfrentamos problemas en una sola dimensión, la debemos entender en su conjunto o de forma multidimensional, lo cual se concibe en un análisis integral del territorio basado en un componente o dimensión económica, ambiental, social y político institucional.

De todo esto se desprende finalmente el marco conceptual de la Reserva de Biosfera que en la diversa literatura su acepción está ligada a una herramienta para el ordenamiento territorial, como un modelo, un territorio, lugares especiales para las personas, entre otras expresiones. (Bioret, Cibien, Grénot, y Lecomte, 1998; Moreira y Borsdorf, 2014). Precisando de una vez, la definición oficial deviene del Marco Estatutario de la Red Mundial de Reservas de Biosfera (a través de la Resolución 28 C/2.4), documento del Programa El Hombre y la Biosfera (MAB por sus siglas en inglés) UNESCO (1995) puntualiza en su Art. 1. "zonas de ecosistemas terrestres o costeros/marinos, o una combinación de los mismos, reconocidas en el plano internacional (...)" (p.17), cabe decir, el Marco Estatutario además define tres funciones de la Reserva de Biosfera, a decir: conservación, desarrollo y apoyo logístico a la investigación, así mismo, de acuerdo a sus criterios para su cumplimiento se basa en un sistema de zonificación marco establecido: área/as núcleo, área/as de amortiguamiento, y área de transición, estas tienen una relación univoca con sus funciones.

\section{Metodología}

Los aspectos metódicos versan en dos partes, se precisa en un inicio las técnicas y los instrumentos que el autor empleo para la construcción de información estadística de línea base para el territorio del ABMC. En segunda instancia se aborda los pasos metodológicos de análisis multidimensional para la estimación del nivel de desarrollo sostenible de los territorios rurales a través del Índice de Desarrollo Sostenible.

\section{Construcción de información base}

En este propósito se toma como punto de partida la información del (último) VII Censo de Población y VI de Vivienda (CPV) del año 2010, en argumentación el autor sostiene que esta operación estadística muestra información confiable para Divisiones Administrativas Menores (DAME) como el caso de las parroquias rurales, sobre el tema, Fox (2014) declara, "the Population and Housing Census is often the only source of valuable informatión needed for planning and management, and research" (p.6)

\section{Procedimiento metodológico para obtención de in- formación base en la reserva de biosfera}

1) Representación espacial de información 


\section{Insumos:}

- Shape del Área de Biosfera Macizo del Cajas (perímetro de la reserva de biosfera y nivel de sistemas de zonificación Núcleo, Amortiguamiento y Transición).

- Cartografía censal del INEC: puntos de localidades en el sector disperso y polígono a nivel amanzanado.

Actividad 1.1 Proceso de espacialización de variables usando herramientas de geoprocesamiento o análisis espacial.

\section{Producto 1.1}

- Homogenización de la representación espacial de los datos (válido para las bases estadísticas de población, hogar y vivienda) en el ABMC y la zonación marco establecida (núcleo, amortiguamiento, transición) ejecutando o haciendo uso de herramientas de geoprocesamiento para obtener centroides de los polígonos de las manzanas (sector amanzanado), obteniendo así una geometría de puntos similar a la existente en el sector disperso, (Ver Figura 1).

- Del proceso resultante se obtiene la base de datos plana (formato .dbf) del ABMC con variables principales: DPA_LOCALIDAD; DPA_MANZAN; TIPO, entre otras. En su orden, las dos primeras presentan un código numérico de 14 caracteres, de estos los seis primeros identifican (provincia, cantón, parroquia urbana y rural) la División Política Administrativa (DPA), y de levantamiento censal utilizado por el INEC; la variable categórica de escala nominal TIPO identifica los puntos (localidades) en las zonas núcleo, amortiguamiento y transición del ABMC, (Ver Tabla 1).

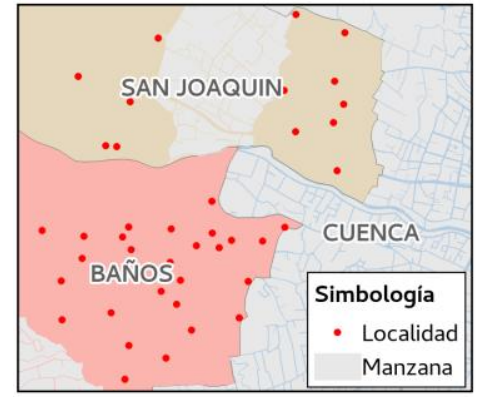

Georreferenciación de localidades

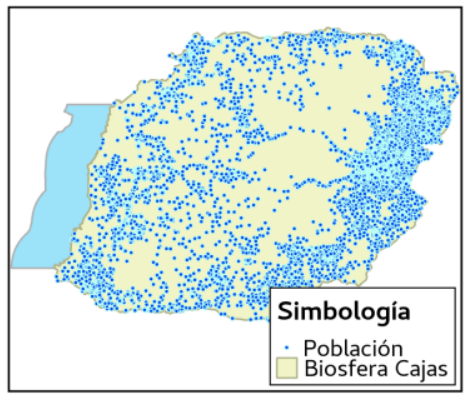

Distribución de la población

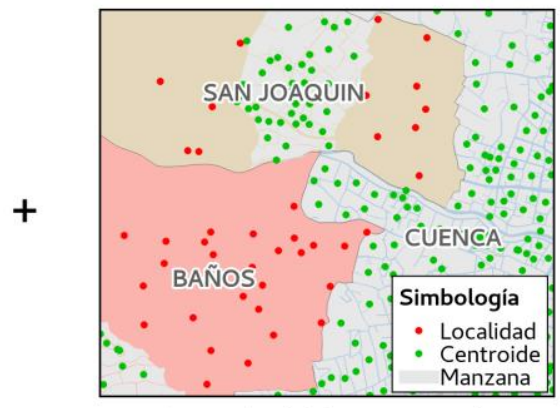

Centroides del área amanzanada

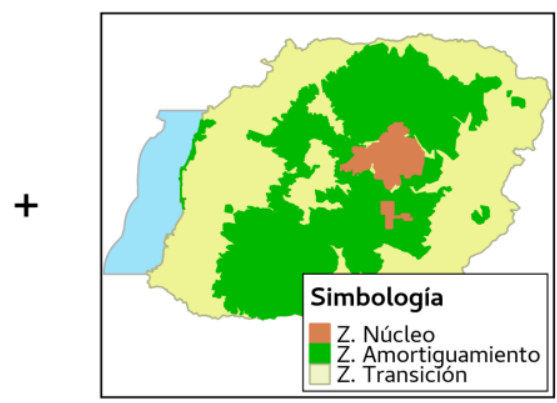

Zonificación ABMC

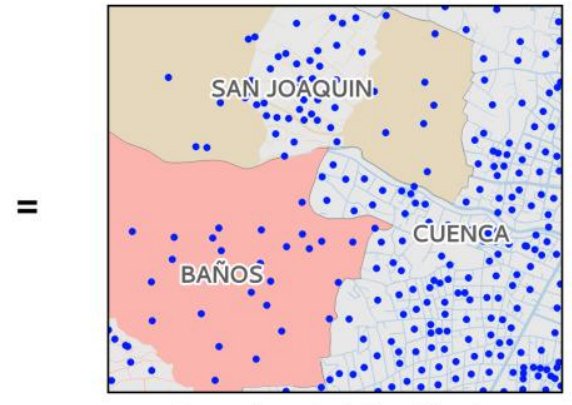

Espacialización de la población

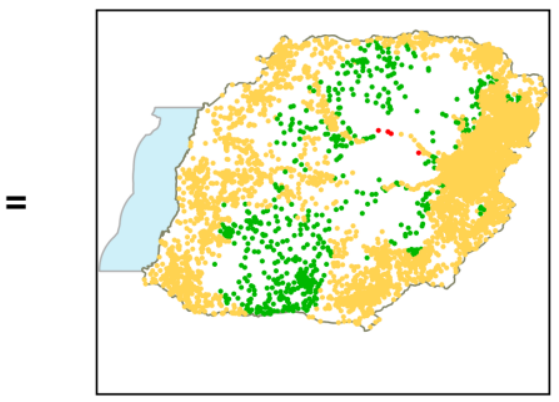

Distribución de la población por zonas

Figura 1. Proceso de espacialización1 de variables usando herramientas de geoprocesamiento o análisis espacial

Fuente: Cartografía censal Instituto Nacional de Estadística y Censos (INEC); Cartografía base Instituto Geográfico Militar (IGM), Patrimonio de Áreas Naturales del Estado (PANE), Ministerio del Ambiente del Ecuador (MAE).

Elaboración: el autor

Nota: En el área dispersa la población se encuentra representada espacialmente mediante una geometría de puntos, denominada localidades. En el área amanzanada la población se encuentra representada en una geometría de polígonos, denominada manzanas. 
Tabla 1 Base de datos planal del Área de Biosfera Macizo del Cajas, y a nivel de Zonificación núcleo amortiguamiento y transición

\begin{tabular}{|c|c|c|c|c|c|c|c|}
\hline DPA_LOCALI & DPA_ANIO & DPA_SECDIS & DPA_DESLOC & PRINCIPAL & TIPO & DPA_MANZAN & DPA_SECTOR \\
\hline 01035899900106 & 2010 & 010358999001 & BURASHUN (TEPAL) & & TRANSICION TERRESTRE & & \\
\hline 01065099900401 & 2010 & 010650999004 & MACARENA ALTO & & AMORTIGUAMIENTO TERRESTRE & & \\
\hline 01065099900402 & 2010 & 010650999004 & COCHAS & & AMORTIGUAMIENTO TERRESTRE & & \\
\hline 01065099900403 & 2010 & 010650999004 & PARSHI (MACARENA) & & AMORTIGUAMIENTO TERRESTRE & & \\
\hline 01065099900404 & 2010 & 010650999004 & LAUREL & & AMORTIGUAMIENTO TERRESTRE & & \\
\hline 01065099900203 & 2010 & 010650999002 & LAS NIEVES & 1 & AMORTIGUAMIENTO TERRESTRE & & \\
\hline 01065099900204 & 2010 & 010650999002 & SAN JUAN PAMBA & & AMORTIGUAMIENTO TERRESTRE & & \\
\hline 01065099900205 & 2010 & 010650999002 & TUCTO & & AMORTIGUAMIENTO TERRESTRE & & \\
\hline 01075099900806 & 2010 & 010750999008 & SAN ISIDRO & 1 & TRANSICION TERRESTRE & & \\
\hline 01075099900807 & 2010 & 010750999008 & CUSCOPALTE & & TRANSICION TERRESTRE & & \\
\hline \multirow[t]{10}{*}{01075099900808} & 2010 & 010750999008 & SUCOS & & TRANSICION TERRESTRE & & \\
\hline & 0 & & & & TRANSICION TERRESTRE & 03035500100105 & 2010 \\
\hline & 0 & & & & TRANSICION TERRESTRE & 03015000800801 & 2010 \\
\hline & 0 & & & & TRANSICION TERRESTRE & 03015400100301 & 2010 \\
\hline & 0 & & & & TRANSICION TERRESTRE & 03015400100316 & 2010 \\
\hline & 0 & & & & TRANSICION TERRESTRE & 03015000801210 & 2010 \\
\hline & 0 & & & & TRANSICION TERRESTRE & 03015500100104 & 2010 \\
\hline & 0 & & & & TRANSICION TERRESTRE & 03015500100105 & 2010 \\
\hline & 0 & & & & TRANSICION TERRESTRE & 03015000701004 & 2010 \\
\hline & 0 & & & & TRANSICION TERRESTRE & 03015000701005 & 2010 \\
\hline
\end{tabular}

Fuente: Cartografía censal Instituto Nacional de Estadística y Censos (INEC); Cartografía base Instituto Geográfico Militar (IGM), Patrimonio de Áreas Naturales del Estado (PANE), Ministerio del Ambiente del Ecuador (MAE).

Elaboración: el autor

Nota: (1) se expone una parte de la base de datos resultado del proceso de espacialización de variables (la base contempla un total de 14.533 registros o casos). Nótese que la base de datos muestra información de identificación de localidades, pero no registra ningún tipo de estadística censal.

2) Construcción de base de datos con información de población, hogares, vivienda del ABMC y a nivel de zonificación: Por conveniencia, la siguiente matriz explica en síntesis la construcción de información base.

Tabla 2

\begin{tabular}{|c|c|}
\hline Paquete de trabajo y actividades & Producto \\
\hline \multicolumn{2}{|c|}{ Construcción base de datos a nivel del ABMC y a niveles de la zonación marco establecida } \\
\hline $\begin{array}{l}\text { Actividad 1.1 Selección de casos mediante criterios condicionales Mediante } \\
\text { criterios condicionales se selecciona subconjuntos de casos de bases oficiales } \\
\text { del INEC (población, hogar, vivienda), esto es unidades territoriales identifi- } \\
\text { cadas (Refiérase Figura 3.2) en el ABMC, respetando el orden de la DPA. }\end{array}$ & \multirow{6}{*}{$\begin{array}{l}\text { Base de datos con información estadística } \\
\text { (población, hogares, y vivienda) del territorio } \\
\text { del Área de Biosfera Macizo del Cajas } \\
\text { (ABMC) y a nivel zonas de planificación } \\
\text { marco establecidas: núcleo, amortiguamiento } \\
\text { y transición }\end{array}$} \\
\hline $\begin{array}{l}\text { Actividad 1.2 Selección de subconjuntos (sectores censales de pertenencia } \\
\text { a las parroquias, y áreas de la Biosfera) Sintaxis de aplicación en partición } \\
\text { de sectores de base matriz (parroquias). De forma análoga a nivel cantonal u } \\
\text { otra división administrativa menor. }\end{array}$ & \\
\hline $\begin{array}{l}\text { Actividad 1.3 Creación variable al nuevo conjunto de datos. Introducción } \\
\text { de la variable denominada SUBSE (SUBSECTOR/MANZANA) al nuevo } \\
\text { conjunto de datos, con sus respectivos datos numéricos. }\end{array}$ & \\
\hline $\begin{array}{l}\text { Actividad. 1.4 Selección de subconjuntos (subsectores de pertenencia a } \\
\text { sectores censales en función de las zonas de la Biosfera. }\end{array}$ & \\
\hline $\begin{array}{l}\text { Actividad 1.5 Fundición de bases (añadir casos) Sintaxis de aplicación en } \\
\text { fundir sectores y subsectores para la construcción de un nuevo conjunto de } \\
\text { datos, parroquias en función de las zonas de la Biosfera. Fundir bases de } \\
\text { parroquias rurales de pertenencia a } \mathrm{N} \text { cantón; fundir bases de cantones de } \\
\text { pertenencia a } \mathrm{N} \text { provincia; y fundir bases de provincia de pertenencia a las } \\
\text { zonas de biosfera. Finalmente se procede a fundir las bases a nivel de cada } \\
\text { una de las tres zonas para tener la base del ABMC. }\end{array}$ & \\
\hline $\begin{array}{l}\text { Actividad. 1.6 Cálculo de indicadores y contraste con base matriz Recuento } \\
\text { de variables a nivel de zonificación. Contraste de conteos de totales a } \\
\text { diferentes unidades. }\end{array}$ & \\
\hline
\end{tabular}

\subsection{Metodología de estimación del desarrollo sostenible}

El enfoque metodológico se guía en todo momento a través de los diversos documentos desarrollados por Sepúlveda et al. (2008) con énfasis en la metodología para estimar el grado de desarrollo sostenible en los territorios.

Sobre estas bases, seguidamente explicamos los pasos metodológicos que guían la investigación para la estimación del nivel de desarrollo sostenible de territorios rurales en el ABMC.

\subsection{Selección de la unidad de análisis}

La definición del área de estudio contempla al territorio denominado Área de Biosfera Macizo del Cajas (ABMC), que abarca y se construye a la vez por gobiernos subnacionales de diferentes niveles (provincial, cantonal y parroquial rural) de acuerdo al perímetro del área, lo que resulta en cortes tangenciales, dicho de otro modo, no contempla a toda la provincia y la contención de todos sus cantones. 
Con referencia a lo anterior, para la estimación del nivel de desarrollo sostenible, el autor selecciona como unidad básica de observación y análisis (UA) a 51 territo- rios parroquiales rurales en donde los Gobierno Autónomos Descentralizados Rurales realizan su gestión junto a otros niveles en el territorio.

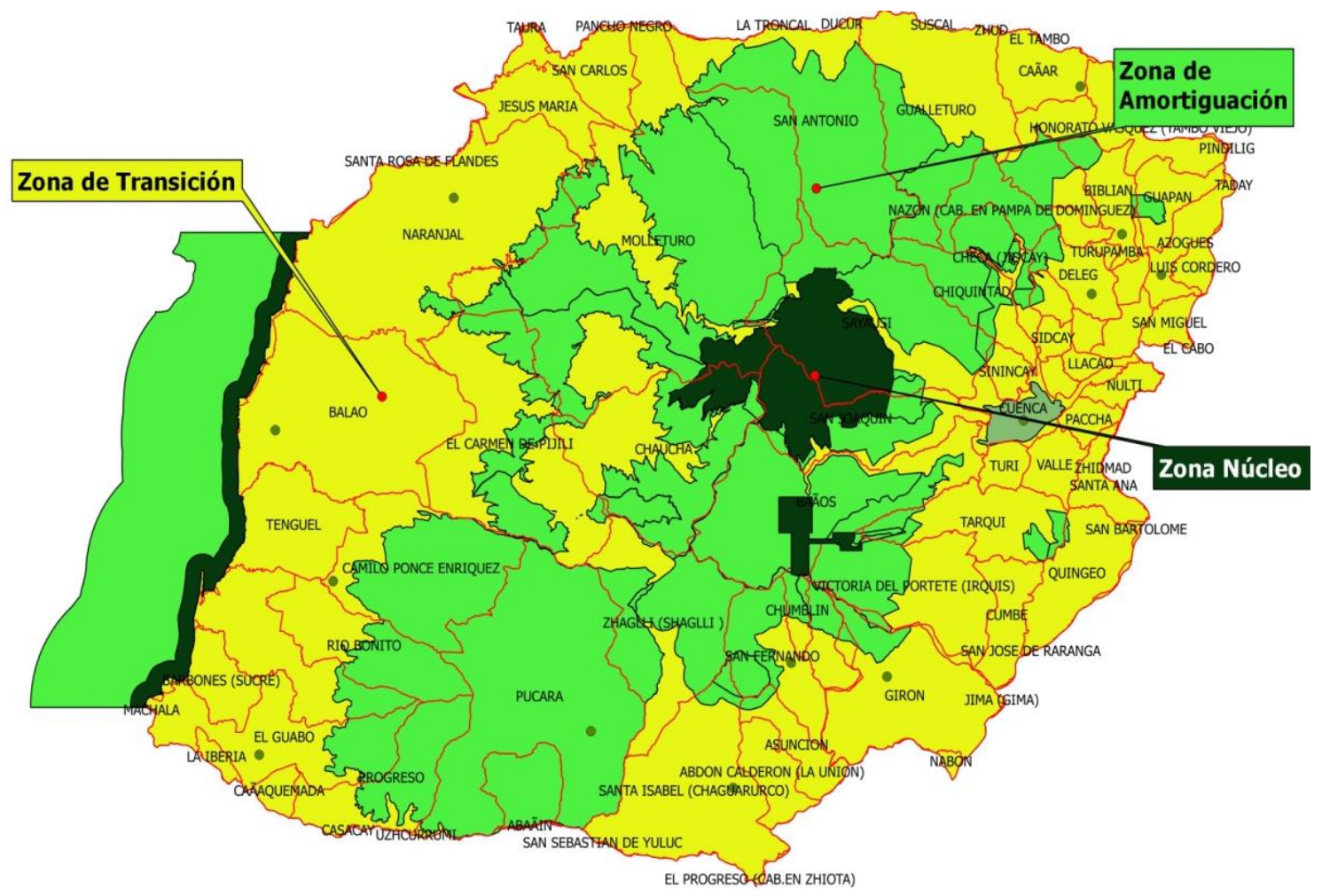

Fuente: Cartografía censal Instituto Nacional de Estadística y Censos (INEC); Cartografía base Instituto Geográfico Militar (IGM), Patrimonio de Áreas Naturales del Estado (PANE), Ministerio del Ambiente del Ecuador (MAE).

Elaboración: el autor

\subsection{Temporalidad}

Por lo que se refiere a la definición del tiempo (t), la investigación contempla data de corte transversal al año 2010 que responde a la construcción de información de línea base, que permite contrastar diferentes territorios en el mismo momento temporal, sin embargo, como se verá más adelante y de acuerdo a la disponibilidad de la información se sugiere consideraciones técnicas de las fuentes de información para suplir vacios de información en esa temporalidad.

\subsection{Definición de dimensiones e indicadores}

De un total de 113 indicadores $^{2}$ por categorías establecidas, otros índices compuestos como las Necesidades
Básicas Insatisfechas (NBI) y entre otros indicadores para las dimensiones ambiental y económica se identificó en orden de prelación la máxima expresión para analizar las dimensiones. Se enfatiza que la base de datos del ABMC construida objetivo específico de la investigación serán el insumo principal, pero no la única fuente de información para la operacionalización de estos indicadores.

En este propósito, a continuación en la Tabla 3 se presentan los indicadores seleccionados y construidos por cada dimensión. Sepúlveda et al. (2008) afirma. "el significado de un indicador va más allá que lo que realmente mide, y más bien representa un fenómeno de mayor trascendencia" (p. 31).

\footnotetext{
${ }^{2}$ Matriz de Indicadores Censales, http://www.ecuadorencifras.gob.ec/manejo-de datos/
} 
Tabla 3 Indicadores para medir el nivel de desarrollo sostenible, según Dimensiones

\begin{tabular}{|c|l|}
\hline Dimensión & \multicolumn{1}{c|}{ Indicadores } \\
\hline Social & $\begin{array}{l}\text { Índice de acceso a servicios públicos básicos; Pobreza por Necesidades Básicas Insatisfechas (NBI); Tasa de embarazo } \\
\text { adolescente (Mujeres adolescentes con hijos en el año censal); Tasa de mortalidad general (t)a, Tasa de Analfabetismo; } \\
\text { Escolaridad promedio de la población femenina; Capacidad de atracción territorial reciente. }\end{array}$ \\
\hline Económica & $\begin{array}{l}\text { Grado de ocupación de la población; Empleo en rubros no primarios (servicios e industria); Actividades realizadas dentro del } \\
\text { hogar; Pobreza por consumo; Gini de consumo; Esfuerzo administrativo; Ejecución presupuestaria de la inversión; Accesibilidad } \\
\text { a zonas homogéneas. }\end{array}$ \\
\hline Ambiental & $\begin{array}{l}\text { Proporción de hogares que utiliza combustibles sólidos para cocinar; Viviendas según forma de eliminación de la basura; } \\
\text { Relación entre zonas protegidas para mantener la diversidad biológica y la superficie total; Tasa de deforestación; Conflicto } \\
\text { en el uso de la tierra; Conectividad Ecológica; Fragmentación del paisaje. }\end{array}$ \\
\hline
\end{tabular}

Fuente: Matriz de indicadores censales, INEC, CPV 2010, Estadísticas Vitales (Nacimientos y Defunciones), Indicadores para la Caracterización y Ordenación del Territorio, Proyecto SIGTIERRAS,

Elaboración: el autor

Nota: ): (a) corresponden a las defunciones ocurridas en el año de estudio e inscritas en el mismo año de investigación.

\subsection{Relación}

Ciertamente los indicadores implican relaciones positivas o negativas en cuanto al estado de desarrollo sostenible, por ello, la metodología plantea definir la posición respecto al bienestar del sistema. En efecto, a medida que el comportamiento del indicador aumente, el estado de cada dimensión o del desarrollo sostenible en sí mismo resultara mejor o peor. En la Tabla 4 se denota la señal (positiva/negativa) de los indicadores en cada dimensión.

\subsection{Transformación de indicadores en los índices}

Dadas las condiciones que anteceden respecto a la posición de cada signo, estos indicadores presentan unidades de medida no comparables, para ello es imperativa la conversión en índices para la agregación de las dimensiones. En definitiva y ya explicado en la metodología se opta por ajustar el valor observado o primario de cada indicador a una escala común siendo el valor mínimo 0 (cero) y el valor máximo 1 (uno), proceso que acopla esta diversidad de indicadores y unidades de medida creando el estado deseable para el agregado en las tres dimensiones (social, ambiental y económico).

La estandarización de las unidades de medida en los indicadores por dimensión utilizando la función de relativización indica mejor y peor situación del sistema con valores entre 1 (uno) y 0 (cero) respectivamente.

Tabla 4 Indicadores para medir el nivel de desarrollo sostenible, según Dimensiones

\begin{tabular}{|c|c|}
\hline Indicador & Relación \\
\hline Índice de acceso a servicios públicos básicos & \\
\hline Escolaridad promedio de la población femenina. & \\
\hline Capacidad de atracción territorial reciente & \\
\hline Grado de ocupación de la población. & \\
\hline Empleo en rubros no primarios. & \\
\hline Actividades realizadas dentro del hogar. & \\
\hline Esfuerzo Administrativo. & + \\
\hline Ejecución presupuestaria de la inversión. & + \\
\hline Accesibilidad Territorial a zonas homogéneas (Accesibilidad elevada). & \\
\hline Relación entre zonas protegidas para mantener la diversidad biológica y la superficie total. & \\
\hline Conflicto en el uso de la tierra (Uso Adecuado/Sin conflicto). & \\
\hline Viviendas según forma de eliminación de la basura. & \\
\hline Conectividad ecológica & \\
\hline Fragmentación del Bosque (área de bosque que está en cada categoría: núcleo). & \\
\hline Pobreza por Necesidades Básicas Insatisfechas (NBI). & \\
\hline Tasa de embarazo adolescente. & \\
\hline Tasa de mortalidad general (t). & \\
\hline Tasa de Analfabetismo. & \\
\hline Pobreza por consumo. & \\
\hline Coeficiente de Gini de consumo. & - \\
\hline Accesibilidad Territorial a zonas homogéneas (Accesibilidad reducida). & \\
\hline Porcentaje Hogares que Utilizan Combustibles Sólidos. & \\
\hline Tasa de deforestación. & \\
\hline Conflicto en el uso de la tierra (Subutilizado; Sobreutilizado/Con conflicto). & \\
\hline Fragmentación del Bosque (área de bosque que está en cada categoría: perforado, borde, parche, transición, áreas agrícolas y pa & \\
\hline
\end{tabular}

Fuente: Matriz de indicadores censales, INEC, CPV 2010, Estadísticas Vitales (Nacimientos y Defunciones), Indicadores para la Caracterización y Ordenación del Territorio, Proyecto SIGTIERRAS,

Elaboración: el autor

Nota: ): (a) corresponden a las defunciones ocurridas en el año de estudio e inscritas en el mismo año de investigación. 


\section{Resultados}

\subsection{Cálculo del índice de desarrollo sostenible: Área de Biosfera Macizo del Cajas}

Los resultados del análisis, más que cifras frías, develan a los territorios rurales que insertos a uno de consideraciones especiales (área de biosfera) presentan sus propias "dolencias". Hecha esta salvedad, el Territorio de la Biosfera en donde por definición pugna el Desarrollo Sostenible es testeado en la dimensión social, económica y ambiental sobre la base de 26 indicadores, encontrando un territorio con nivel de estado del sistema inestable, atribuido por un IDS de $\mathbf{0 , 5 1}$. Las tres dimensiones referidas de forma específica representan un estado de inestabilidad. En la Tabla 5 se presentan los índices específicos.

Tabla 5 Índice de desarrollo sostenible y por dimensiones del Área de Biosfera Macizo del Cajas

\begin{tabular}{|c|c|c|c|c|}
\hline \multirow{2}{*}{ Calculo } & \multicolumn{3}{|c|}{ Dimensiones de análisis } & $\begin{array}{c}\text { IDS } \\
\text { territorio } \\
\text { Biosfera }\end{array}$ \\
\cline { 2 - 4 } & Social & Económica & Ambiental & \\
\hline $\begin{array}{c}\text { IDS promedio } \\
\text { Aritmética }\end{array}$ & 0,48 & 0,51 & 0,51 & 0,50 \\
\hline $\begin{array}{c}\text { IDS promedio } \\
\text { Armónica. }\end{array}$ & 0,44 & 0,45 & 0,44 & 0,45 \\
\hline
\end{tabular}

Elaboración: el autor, sobre la base de los datos de cada una de las dimensiones construidas.

En efecto de forma general el $80 \%$ de las 51 unidades territoriales tienen un nivel de estado del sistema inestable, en la categoría de sistemas estables encontramos a un $12 \%$, y finalmente el porcentaje restante ubica a las parroquias de Gualleturo, Quingeo, San Rafael de Zharug y Turupamba con nivel de estado del sistema critico, atribuido al desequilibrio entre dimensiones y de los indicadores dentro de cada dimensión, teniendo en cuenta que los indicadores no tienen la misma señal a los procesos de desarrollo. La Figura 3 muestra el nivel del estado del sistema.

Figura 3. Nivel de estado del sistema según territorios rurales del Área de Biosfera Macizo del Cajas

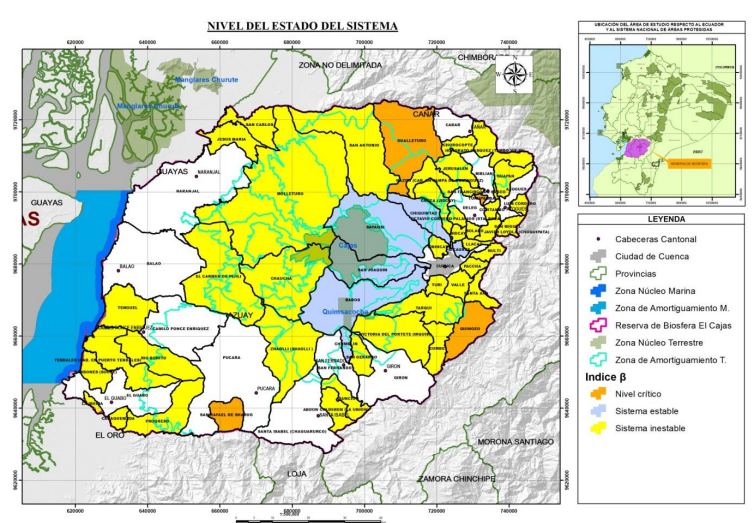

Elaboración: el autor

\section{Conclusiones}

Distinguimos tres grados de desarrollo diferenciados entre los territorios parroquiales rurales de la Biosfera del Cajas. Para empezar, encontramos a las parroquias de San Rafael de Sharug, Gualleturo, Quingeo y Turupamba que evidencian el nivel crítico del sistema (rango mayor que 0,2 y menor que 0,4 de su IDS), debiendo sortear las dificultades de rezago debiendo poner énfasis en el acceso a servicios básicos, hogares en hacinamiento, y la educación de la mujer rural; por el lado de la dimensión económica la atención directa a mejorar la estructura económica del territorio en lo referente al empleo, En efecto las cifras revelan que en Gualleturo apenas el 7,6\% de la PEA ocupada tiene un empleo cercano a tiempo completo, lo que se traduce en menor desarrollo económico

En segundo lugar tenemos al grupo conformado por 41 territorios rurales con un nivel de estado del sistema inestable siendo el valor del IDS entre 0,40 y 0,6 . El desequilibrio entre las dimensiones es más complejo de acuerdo a las realidades territoriales de cada parroquia. Finalmente los territorios rurales de Baños, Chiquintad, Ricaurte, San Joaquín, Sayausi y Chumblin todos de la provincia del Azuay tienen un nivel de estado del sistema catalogado como estable, esta categoría se traduce en un matiz de potencialidades en cada dimensión, sin embargo se encuentra todavía rezagos.

A manera de colofón los patrones de desarrollo dependen de la definición de rural, territorios rurales cercanas a ciudades son más dinámicas que los territorios rurales alejados, esto da cuenta de la tendencia geográfica del indicador de empleo en rubros no primarios, las diferencias entre los territorios rurales de San Joaquín, Santa Ana, Sayausi, Chiquintad, Baños, Paccha, Nulti, Llacao, Sinincay, Valle, Turi y Ricaurte cuya población esta empleada en más del $75 \%$ al sector industrial y de servicios (desde el punto de vista territorial responde a la accesibilidad y cercanía a la ciudad de Cuenca por su perfil económico y gran núcleo urbano), y los territorios de Zhaglli, El Carmen de Pijilí, Cañaquemada, Gualleturo, Progreso, Jerusalén, Tendales, Barbones, Sharug, San Antonio, San Carlos, Chaucha, Nazon, Rio Bonito en donde el empleo de la población es menor en los sectores de servicios e industria (entre $16 \%$ y $30 \%)$.

Por lo que se refiere a objetivos específicos como gradientes hacia la consecución del objetivo final comentamos las siguientes conclusiones:

- Base de datos con información estadística de población, hogares, y vivienda propia del territorio del Área de Biosfera Macizo del Cajas (ABMC) y a nivel de zonas de planificación marco establecidas: núcleo, amortiguamiento y transición mediante un proceso de espacialización de variables usando herramientas de geoprocesamiento o análisis espacial. La contribución a lo establecido en la Estrategia de Sevilla de acuerdo a lo que se considera Indicadores de aplicación que resulta de constituir una base de datos de indicadores 
de sostenibilidad (en términos ecológicos, económicos, sociales, entre otros).

- Con referencia a lo anterior se tiene evidencia empírica del total de población emplazada en relación a la zonificación del ABMC, develando diferencias en valores absolutos y relativos contrastando lo indicado en el documento Expediente de Propuesta, y el Plan de Gestión del Área de Biosfera del Macizo del Cajas (refiérase pág. 55), que desde el punto de vista del OT está arraigado a la población como recurso y sujeto territorial. La inexacta cuantificación de la población repercute en la planificación para el acceso, cantidad, calidad de servicios, infraestructura y equipamiento.

- De la base del ABMC, se utilizó 12 indicadores para la estimación del nivel de desarrollo sostenible.

- Con la información del Sistema de Información para los Gobiernos Autónomos Descentralizados (SIGAD+), SENPLADES se fusiono 51 bases de datos para analizar los problemas del territorio del ABMC, como resultado 1.954 problemas registrados por los GAD parroquiales rurales. La situación del territorio que reflejó por prelación centrar su atención a: Cobertura de servicios básicos, movilidad, vulnerabilidad, clima/agua, capacidad del GAD para la gestión del territorio, entre otros contenidos.

\section{Consideraciones finales y recomendaciones}

No existen soluciones mágicas o policías públicas que no se hayan hecho, basta con no seguir haciendo más de lo mismo. La receta desde el criterio y experiencia del autor es la multiescalaridad, es decir, la articulación entre diferentes niveles de gobierno para encontrar solución al desarrollo, se recuerda que se planifica en un mismo territorio no para una parte de la población o superficie de esta.

No podemos seguir hablando por casi 40 años de la falta de acceso a servicios públicos en los sectores rurales cuando es ahí de donde los recursos monetarios salen para la inversión, por atesorar recursos y bienes ambientales para la sostenibilidad, no se está devolviendo la oportunidad que el desarrollo también retorne a ellos, pero la respuesta es, costos elevados, de distancia y baja demanda afecta a la inversión pública y privada. Ante esto para el autor los costos frente a los beneficios de la población rural no están en discusión.

Para el autor la evidencia de una investigación a más de conclusiva se adapta para posteriores análisis, en ese sentido la metodología como otras de potencial deberían ser insumo para la caracterización y ordenación del territorio enmarcado en la fase de diagnóstico y caracterización del sistema territorial.

Al analizar la información para la caracterización de los territorios rurales de la Reserva de Biosfera se encuentra en los PDOT una estructura estándar en cada una de las fases (Diagnostico, Propuesta y Modelo de Gestión) y para cada nivel de gobierno subnacional, sobre lo cual se recomienda a las entidades de supervisión y entes de la planificación diferenciar contenidos y estructura de acuerdo a las competencias de los GAD parroquiales rurales, dado que su camino de desarrollo es sustancialmente diferente a los modelos cantonal o provincial.

Se recomienda a los entes encargados de la planificación, el ordenamiento territorial y la información considerar:

- Construir un set de indicadores que cuantifique o mida el comportamiento del sistema territorial dentro de los componentes biofísicos, sociales y económicos, esto imperativo para dar seguimiento y evaluación a los instrumentos de planificación PDOT, pero ir más allá, la idea no es solo construir indicadores, sino que respondan y sean indicadores aplicables a los estudios de caracterización y Ordenamiento Territorial.

El autor recomienda, considerando los marcos normativos internacionales y que son base de una reserva de biosfera, utilizar a las bases de datos resultantes como información de línea base para el ABMC:

- De acuerdo al Art. 9 del Marco Estatutario de la Red Mundial de Reservas de Biosfera, el ABMC deberá ser evaluada en 2023, para saber en qué medida cambio, es imprescindible tener información de línea base, la base construida podrá ser insumo para la toma de decisiones, la idea es que los resultados de la investigación no engrosen una lista de archivos muertos.

- Conviene subrayar que en la taxonomía expuesta sobre instrumentos de planificación y ordenamiento territorial en Ecuador, la Ley ni el Acuerdo figuran como herramienta a los Planes de Gestión de las Reservas de Biosfera, por consiguiente para el autor se considera necesario su inclusión de acuerdo a:

- A lo anterior, el ámbito de su aplicación lo consideramos de acción territorial y supranacional, por un lado su integración a los PDOT y su observancia de las propuestas en cada zona; y por otro lado, por su estructura de vincular espacios transfronterizos (primera Reserva de Biosfera Transfronteriza Binacional de América del Sur entre Ecuador y Perú).

- De acuerdo a la definición de los instrumentos citados, el Plan Territorial Diferenciado se acopla a la concepción de Reserva de Biosfera, en la actualidad tenemos casos específicos: Plan de Desarrollo Sustentable de Galápagos (PDSOT), Plan Integral Amazónico (PIA), Plan para el Desarrollo Sostenible del Espacio Marino Costero (PDSEMCE), ahora bien, para el autor, si bien se encuentran en concepciones idénticas por la integración del territorio y por las condiciones biofísicas y socioculturales, la diferencia en su aplicación conviene a que en las Reservas de Biosfera la yuxtaposición de su perímetro corta unidades territoriales, en contraposición los PTD contemplan regiones completas.

\section{Referencias Bibliográficas}

Bioret, F., Cibien, C., Grénot, J.-C., y Lecomte, J. (1998). Una guía para la gestión de reservas de biosfera: 
una metodología aplicada a las reservas de biosfera francesas. compendio mab 19 (1ra ed.). Montivedio, UR. (UNESCO para América Latina y el Caribe)

Castro Riera, C. (2015). Normativa constitucional, legal y reglamentaria vinculada a la ordenación territorial. Cuenca.

Fox, K. (2014). The use of census data for national development planning: Focus on the 2010 population and housing census. Chile, $\mathrm{CH}$ : United Nations.

Gómez, D., y Gómez, A. (2013). Ordenación territorial (3ra. ed.). Madrid: Mundi-Prensa.

Moreira, A., y Borsdorf, A. (2014). Reservas de la biosfera de chile: Laboratorios para la sustentabilidad (1 ra ed.). Santiago, CH: Geolibros.

Schneider, S., P., W., Filippi, E., Ruckert, A., Rambo, A., Radowsky, G., ... Spethc, S. (2007). Análisis multidimensional y escalar del desarrollo territorial en brasil. FAURGS.

Sepúlveda, S. (2008). Biograma: metodología para estimar el nivel de desarrollo sostenible de territorios (1ra ed.). San José.

UNESCO. (1995). Reservas de biosfera: La estrategia de sevilla y el marco estatutario de la red mundial. Paris: Autor.
Recibido: 30 de junio de 2018

Aceptado: 3 de septiembre de 2018

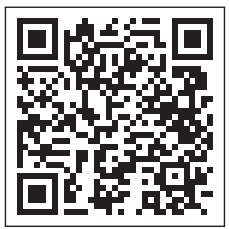

